\title{
Complex Autoinflammatory Syndrome Unveils Fundamental Principles of JAK1 Transcriptional and Biochemical Function
}

\author{
Conor Gruber ${ }^{1}$, Jorg Calis ${ }^{1}$, Sofija Buta ${ }^{1}$, Gilad Evrony ${ }^{2}$, Jerome Martin ${ }^{3,4}$, Skyler Uhl ${ }^{1}$, Rachel \\ Caron $^{1}$, Lauren Jarchin ${ }^{5}$, David Dunkin ${ }^{5}$, Robert Phelps ${ }^{6,7}$, Bryn Webb², Jeffrey Saland ${ }^{2}$, Miriam \\ Merad $^{3,4}$, Jordan Orange ${ }^{10}$, Emily Mace ${ }^{10}$, Brad Rosenberg ${ }^{1}$, Bruce Gelb ${ }^{1,8,9}$, Dusan \\ Bogunovic ${ }^{1,2,3,9, \#}$
}

${ }^{1}$ Department of Microbiology, ${ }^{2}$ Department of Pediatrics, ${ }^{3}$ Precision Immunology Institute, ${ }^{4}$ Department of Medicine, Hematology and Medical Oncology ${ }^{5}$ Department of Gastroenterology, ${ }^{6}$ Department of Dermatology, ${ }^{7}$ Department of Pathology, ${ }^{8}$ Department of Genetics and Genomic Sciences, ${ }^{9}$ Mindich Child Health and Development Institute, Icahn School of Medicine at Mt. Sinai, New York, NY, 10029 United States

${ }^{10}$ Department of Pediatrics, Columbia University Irving Medical Center, New York, NY 10032, United States \#correspondence (lead contact) - Dusan.Bogunovic@mssm.edu

\begin{abstract}
Autoinflammatory disease can result from monogenic errors of immunity. We describe herein the first example of a patient with early-onset widespread autoinflammation resulting from a mosaic, heterozygous, gain-of-function mutation (S703I) in $J A K 1$, encoding a kinase essential for signaling downstream of over twenty-five cytokines. By first-ofits-kind custom single-cell RNA sequencing, we examine mosaicism with single cell resolution. We uncover that JAK1 transcription is predominantly restricted to a single allele across different immune cells, introducing the concept of a mutational "transcriptotype" that differs from the genotype. Functionally, the S703I mutation not only increased JAK1 kinase activity, but also resulted in transactivation of partnering JAKs, independently of its catalytic domain. Further, S703I JAK1 was not solely hypermorphic for cytokine signaling, but neomorphic as well, as it enabled downstream signaling cascades not canonically mediated by JAK1. Given these results, the patient was treated with tofacitinib, a JAK inhibitor, which led to rapid resolution of her clinical disease. Together, these findings represent an unprecedented degree of personalized medicine with the concurrent discovery of fundamental biological principles.
\end{abstract}

\section{Introduction}

Monogenic disease mutations afford the opportunity to study the bona fide function of human genes in vivo, which have guided our understanding of biology and medicine for decades. Undiagnosed disease programs, by means of next-generation sequencing, have recently provided a means to identify, diagnose, and study these rare patients with unusual clinical presentations (Splinter et al., 2018; Lee et al., 2019;
Yang et al., 2019). In turn, clinical management can, in some cases, be highly personalized.

To date, studies of rare immunologic diseases have identified germline gain-of-function (GoF) and loss-of-function (LoF) mutations throughout the JAKSTAT signaling axis (Macchi et al., 1995; Russell et al., 1995; Dupuis et al., 2001; Kofoed et al., 2003; Minegishi et al., 2006, 2007; Holland et al., 2007; Mead et al., 2012; Hambleton et al., 2013; Etheridge et al., 2014) the primary signal transduction pathway for cytokines. The Janus kinase (JAK) family contains four tyrosine kinases (JAK1, JAK2, JAK3, TYK2) constitutively associated with cytokine receptors. Upon cytokine binding, JAKs act in partnership to phosphorylate themselves, the receptors, and then STATs, which can then act directly as transcription factors or activate other signaling pathways further downstream (O'Shea et al., 2015). Uniquely, JAK1 is activated by a remarkably broad range of cytokines (Yc-, gp130-, Interferon- and IL-10-family cytokines). It can phosphorylate any STAT protein (STAT1-6), and is universally expressed in all tissues (O'Shea et al., 2015). Through the formation of specific combinations of cytokine receptors, JAK partners, and STAT dimers, JAK1 orchestrates unique downstream signals for each cytokine.

The need to better understand JAK regulation has deepened with the expanding clinical use of JAK inhibitors (O'Shea and Gadina, 2019). The breadth of successfully treated inflammatory conditions signifies the central pathophysiological role of JAK hyperactivity across immune diseases. Yet, the complete list of disorders resulting from JAK-STAT dysregulation remains unknown. Further, it is unclear which specific JAK-mediated pathways drive disease, a key issue for the design of inhibitors with greater selectivity for individual members of the JAK family. 
Herein, we identify a novel mutation (S703I) of JAK1 in a patient with a severe, early-onset immunodysregulatory syndrome identified in our undiagnosed disease program. Using extensive nextgeneration genomic, molecular and multi-parametric immunological tools, we probe the effects of S703I JAK1 in vitro and ex vivo in order to investigate clinical dysfunction in vivo.

\section{Results \\ Complex immunodysregulatory syndrome}

We studied an 18 year-old female from a nonconsanguineous family who was referred into our undiagnosed disease program with an early-onset, severe syndrome of immune dysregulation. Both her parents and an older brother were healthy (Figure $1 \mathrm{~A})$. In the patient, disease features arose prior to the age of 3 years, and included: diffuse, severe dermatitis; chronic unspecified enteritis and colitis with increased eosinophils; idiopathic membranous nephropathy (MN); fluctuating peripheral eosinophilia; asthma; food and environmental allergies; severely stunted growth with leg length discrepancy and poor weight gain (Figure S1A-E). Of note, serology was negative for known MN autoantibodies. Similarly, neither gastrointestinal nor dermatologic manifestations matched criteria for known diagnostic entities, despite multiple clinical evaluations and biopsies. However, the skin disease was reminiscent of atopic dermatitis, and the linear lesions were similar to those found in inflammatory linear verrucous epidermal nevus (ILVEN). Kidney transplantation was performed at age 11, but MN recurred within one year, followed by antibody-mediated rejection, requiring subsequent hemodialysis. The other inflammatory and autoimmune features were also largely resistant to therapeutic interventions (Supplemental Case Report).

\section{Whole exome sequencing reveals a de novo JAK1 mutation}

Given the overall healthy state of the parents, and the early onset of disease in the patient, we hypothesized that either a recessive or a de novo genetic mutation was causative of the clinical syndrome. We performed whole-exome-sequencing on peripheral blood cells obtained from the patient and her parents. Subsequent variant analysis failed to produce any likely variants by a recessive model of inheritance (Table S1). Given the asymmetric manifestations of disease, including limb-length discrepancy and irregularly distributed dermatitis, we then considered the possibility of lower-read-frequency de novo mosaic mutations, which are typically excluded from common analysis pipelines. One candidate de novo variant, JAK1 c2108G $>$ T, which constituted $27 \%$ of reads mapping to the region, was identified (Figure $1 A-B)$. The presence of the $c 2108 G>T$ variant was confirmed by Sanger sequencing (Figure $1 \mathrm{C}$ ), and this variant was absent from all publicly available genome sequences. This mutation results in substitution of serine to isoleucine at position 703 (S703I) in a highly conserved region (Figure S1F) and is predicted to be highly damaging (CADD score of 27.6). We then investigated the presence of $c .2108 G>T$ in nonhematopoietic tissues. We performed digital droplet PCR (ddPCR) with mutation-specific probes to estimate the fraction of cells carrying the mutation in different tissues. We identified the mutation at various frequencies in DNA from buccal swabs, granulocytes, PBMCs, and endoscopic biopsy samples fractionated into epithelia and associated immune cells (Figure 1D and S1G). These tissues represent all three germ layers, signifying that the mutation must have arisen in the first $\sim 12$ cell divisions between fertilization and gastrulation (Figure 1E)(Moore, Persaud and Torchia, 2015).

\section{Allele characterization indicates that S703I confers a gain-of-function on JAK1}

The S703I mutation localizes to the pseudokinase domain of JAK1, a putative regulatory domain (Figure $2 A)$. Although S703I is positioned adjacent to the two germline JAK1 mutations identified to date, these other mutations diverged in their downstream consequences, making functional predictions for S703I difficult. To assess the possible pathogenicity of the mutation and its impact on JAK1 function, we transduced WT JAK1, S703I JAK1 and empty vector lentiviruses into U4C (JAK1-/-) cells. Transduction with S703I JAK1, but not WT JAK1 or Luciferase, led to basal phosphorylation of STAT proteins and active target gene transcription in the absence of cytokine stimulation (Figure 2B-E). S703I-transduced cells hyper-responded to IFNa, in terms of both the proximal phosphorylation of STAT1 and STAT2 and the induction of interferon-stimulated genes (ISGs) (Figure 2B-C). Similarly, these cells hyperphosphorylated downstream STATs in response to IFNy or IL-6 (Figure 2D-E and S2A).

For direct confirmation of the pathogenicity of the mutation in cells from the patient, we derived an EBV-immortalized B-cell (B-EBV) line from the patient's PBMCs. Given the mosaicism for JAK1 in the patient's cells, individual lines were cloned from single cells to derive purely WT or S703I heterozygous mutant cells (Figure S2B). A comparison of STAT phosphorylation in patient WT and mutant B-EBV cells supported the gain-of-function role of S703I JAK1, both at baseline and in response to cytokine 

aCC-BY-ND 4.0 International license.

A

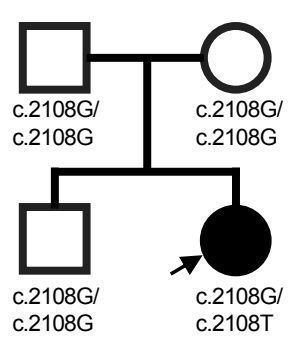

B

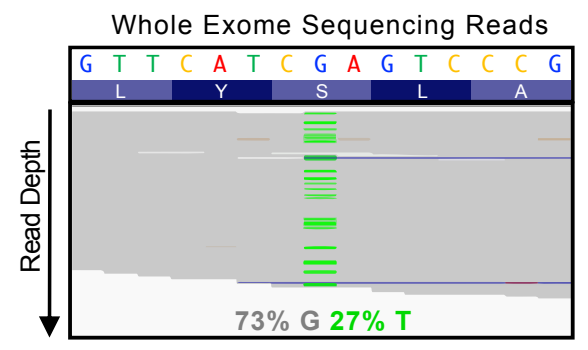

C

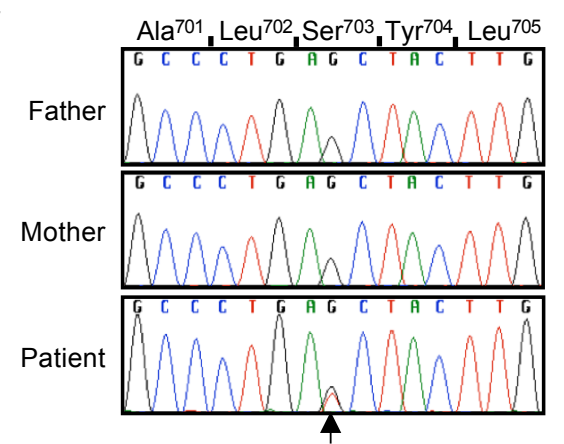

D

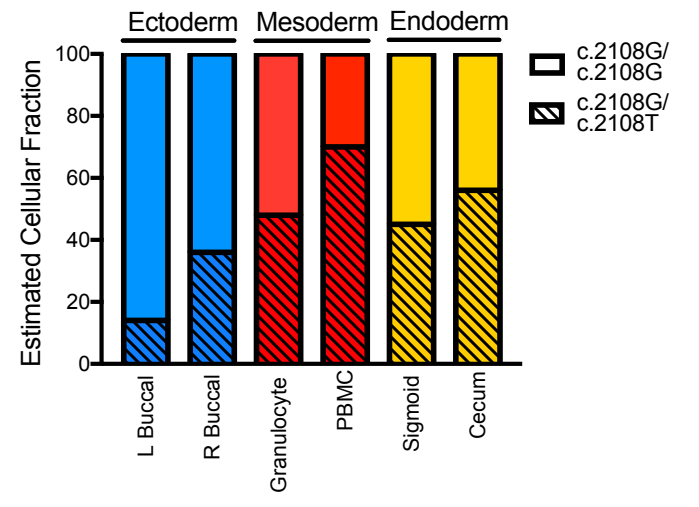

$\mathrm{E}$

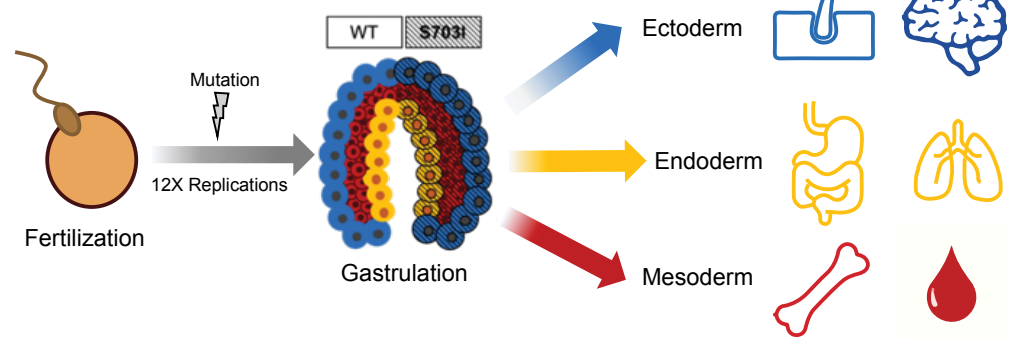

Figure 1. De novo mutation in JAK1 identified in patient with immunodysregulatory syndrome.

(A) Patient's family pedigree. (B) Whole exome sequencing reads mapping to JAK1 locus c.2108 with variant nucleotides displayed in green (C) Representative chromatograms from Sanger sequencing of peripheral blood DNA to confirm c.2108 G>T JAK1 (D) Proportion of cells carrying heterozygous mutation, as estimated by digital droplet PCR with WT- and mutation-specific probes. DNA was obtained from bilateral cheek swabs, Ficoll-fractionated whole blood, and epithelial tissue isolated from a colonic biopsy $(n=1)$. (E) Model for the development of the de novo mutation and its distribution into all three germ layers.

(Figure 2F-G). The isogenic control derived from the same patient definitively identified S703I JAK1 as the probable pathogenic mutation in the patient's genome. Together, these results indicate that S703I is gain-of-function for basal and cytokine-induced STAT signaling.

\section{S703I JAK1 trans-activates partnering JAKs independently of its own kinase activity}

To dissect the mechanisms underlying upregulated STAT signaling, we assessed the impact of S703I on JAK auto-phosphorylation. Consistent with the increase in STAT phosphorylation, S703I JAK1 was itself hyper-phosphorylated (Figure 3A). Interestingly, both JAK2 and TYK2 phosphorylation were also upregulated (Figure 3B-C S2C), suggesting that the interacting JAK partners may also play a role in the gain-of-function. We hypothesized that the mutant JAK1 pseudokinase domain trans-activates the kinase activity of JAK2 and TYK2. To investigate this mechanism, we mutated the ATP-binding site (K908A) of JAK1 to render it catalytically inactive. This well-characterized mutation retains the signaling capability of the receptor complex, making it possible to study signaling by the partnering JAKs in isolation (Li et al., 2013; Eletto et al., 2016). As expected, STAT phosphorylation levels were largely reduced in the absence of JAK1 activity (Figure 3D-E). Yet, remarkably, following inactivation of the kinase domain of S703I JAK1 (S703I/K908A), an aberrant increase in STAT phosphorylation relative to kinaseinactivated JAK1 without the S703I mutation was observed upon cytokine stimulation (Figure 3D-E). This result indicates that S703I JAK1 trans-activates JAK2 and TYK2, revealing that pseudokinase domains can regulate partnering JAKs in trans, in addition to traditionally understood cis-regulation (Babon et al., 2014). This novel mechanism of JAKSTAT signaling regulation, revealed by the rare disease mutation of a single patient, highlights both the potential of rare disease research to discovery of novel physiological mechanisms and the unexpected complexity with which mutations of a kinase gene can enhance signaling independently of their own activity. 
A

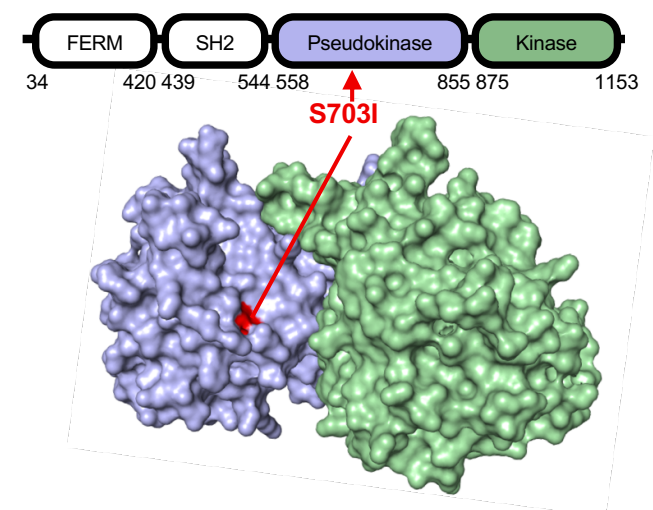

B

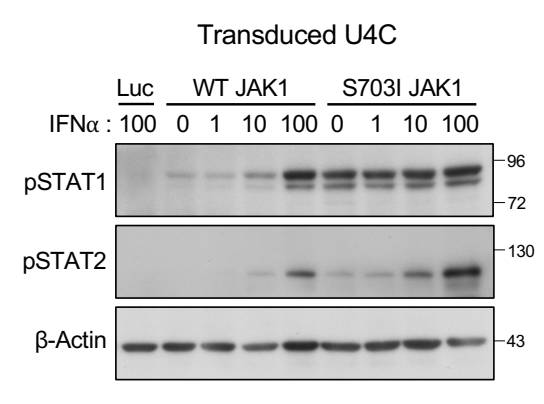

C

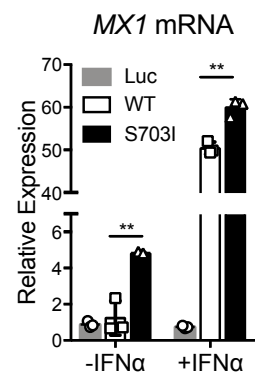

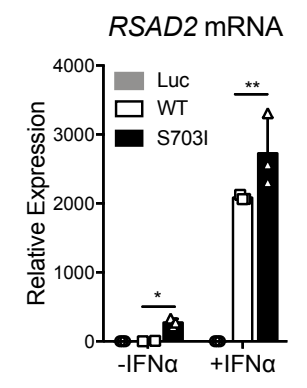

$\mathrm{D}$

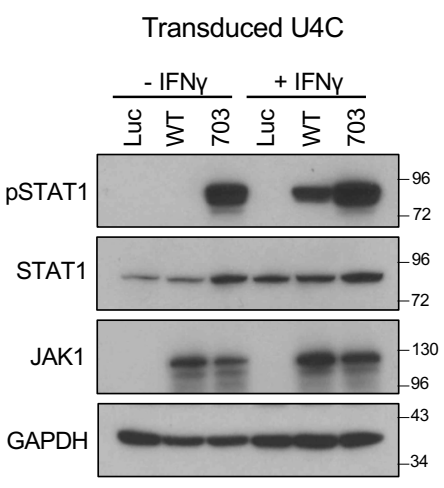

$\mathrm{E}$

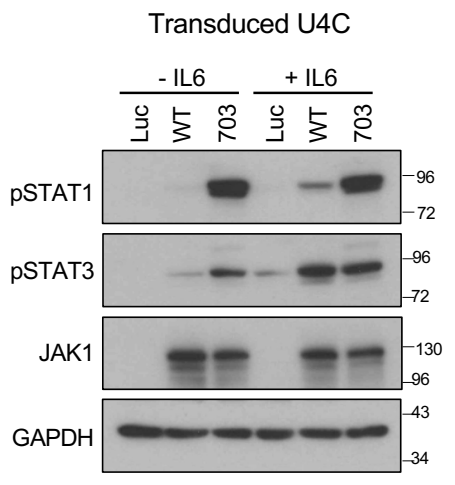

$\mathrm{F}$

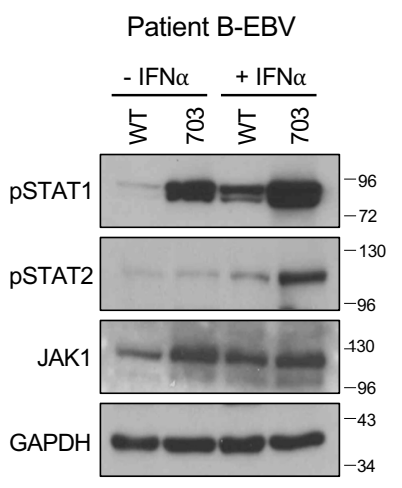

G

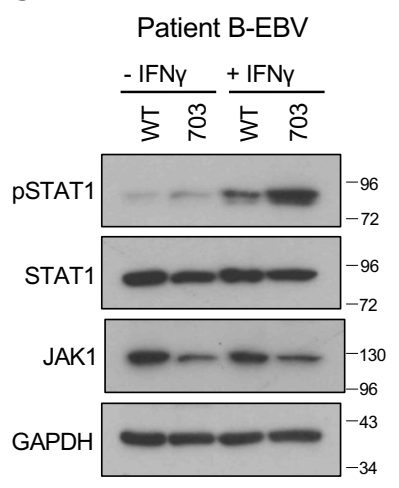

Figure 2. S703I JAK1 confers constitutively active and hyper-responsive STAT signaling.

(A) Localization of S703I (red) mutation to the pseudokinase domain (blue) of JAK1, as represented in the linear sequence and predicted structure modeled on TYK2 (B) Western blotting for STAT phosphorylation in U4C Cells (JAK1-/-) reconstituted with lentiviruses for vector control (Luciferase), WT JAK1, and S703I JAK1 and stimulation for 15 minutes with indicated doses of IFN $\alpha(\mathrm{IU} / \mathrm{mL})$. (C) qPCR from transduced U4C cells for interferon-stimulated gene expression (MX1 and RSAD2) at baseline and following $100 \mathrm{IU} / \mathrm{mL}$ of IFN $\alpha$ for 8 hours (n-3). Columns represent mean and error bars represent standard deviation. ${ }^{*} \mathrm{P}<0.05$. ${ }^{* *} \mathrm{P}<0.01,{ }^{* * *} \mathrm{P}<0.001$, two-tailed Student's $\mathrm{t}$ test with Welch's correction. (D) Stimulation of transduced U4C cells with IFNY $(0.1 \mathrm{ng} / \mathrm{mL})$ or $(E)$ IL6 $(25 \mathrm{ng} / \mathrm{mL})$ for 15 minutes. (F) Derivation of BEBV cells and isolation of JAK1 WT/WT and JAK1 S703I/WT cells from patient blood, followed by stimulation with IFN $\alpha$ $(100 \mathrm{IU} / \mathrm{mL})$ or $(\mathrm{G})$ IFNy $(0.1 \mathrm{ng} / \mathrm{mL})$ for 15 minutes.

\section{Ex vivo analysis reveals an expansion of CD56 ${ }^{\text {hi }}$ NK cells and a cell-intrinsic gain-of-function}

To more robustly investigate the consequences of S703I on immune cells, we performed mass cytometry (CyTOF) immunophenotyping on whole blood from the patient. Surprisingly, despite the central role of JAK1 in immune cell differentiation and proliferation, the patient's immune cell distribution was largely within the normal range, barring a few exceptions (Figure 4A, Table S2). B cells, for instance, trended toward lower frequencies than in healthy donors. Most strikingly though, natural killer (NK) cells exhibited a dramatic expansion of CD56 ${ }^{\text {hi }}$ cells (>12-fold over healthy controls) (Figure 4B, Figure S3A-B). The CD56 ${ }^{\text {hi }}$ subset is the minority population in healthy individuals in whom about $90 \%$ of peripheral blood NK cells are CD56 ${ }^{\text {lo }}$ (Poli et al., 2009). These subsets are broadly defined by their surface density of CD56 but have unique phenotypic and functional properties. Namely, the CD56 ${ }^{\text {hi }}$ subset is less mature but rapidly produces cytokines, whereas the $\mathrm{CD} 56^{\text {lo }}$ subset expresses perforin and granzyme at baseline and mediates cytotoxicity through contact-dependent target cell lysis (Poli et al., 2009). Extensive phenotyping of the patient's NK cells demonstrated that the expanded population retained many prototypical immature features defining them as bona fide "CD56 bright" NK cells (Figure S3C-E). The CD56 ${ }^{\text {lo }} \mathrm{NK}$ cell population was present and similarly 
A

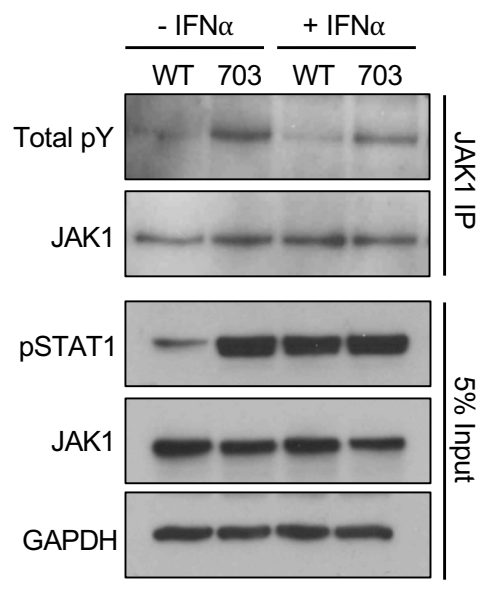

B

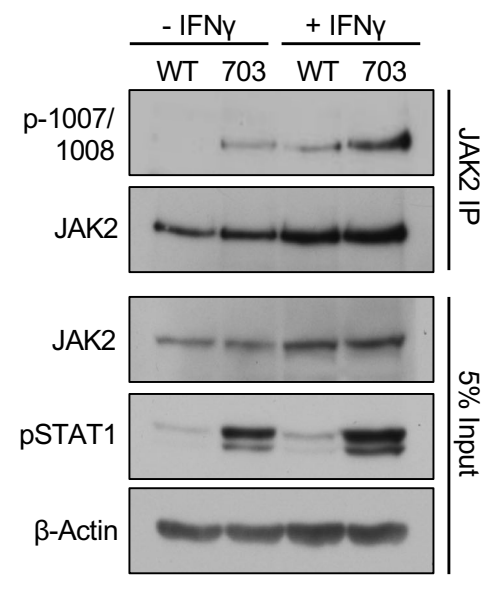

C

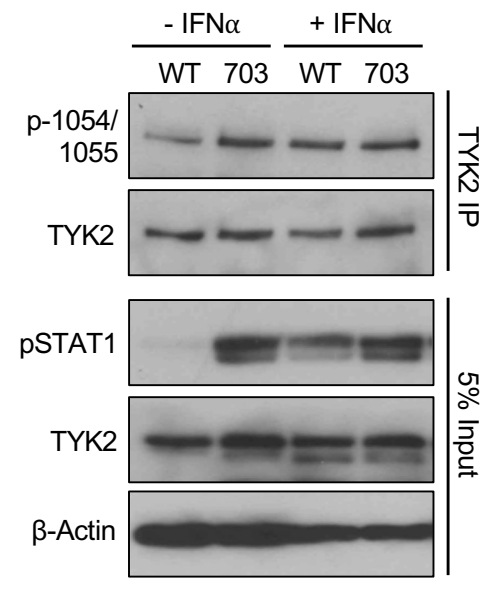

$\mathrm{D}$

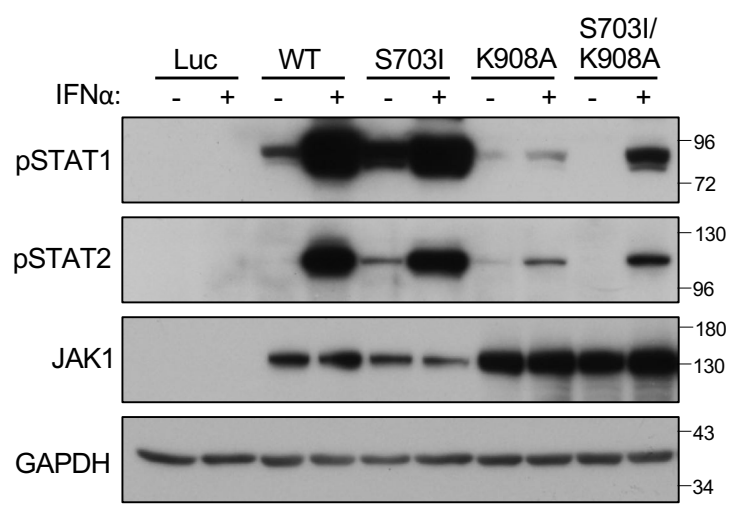

E

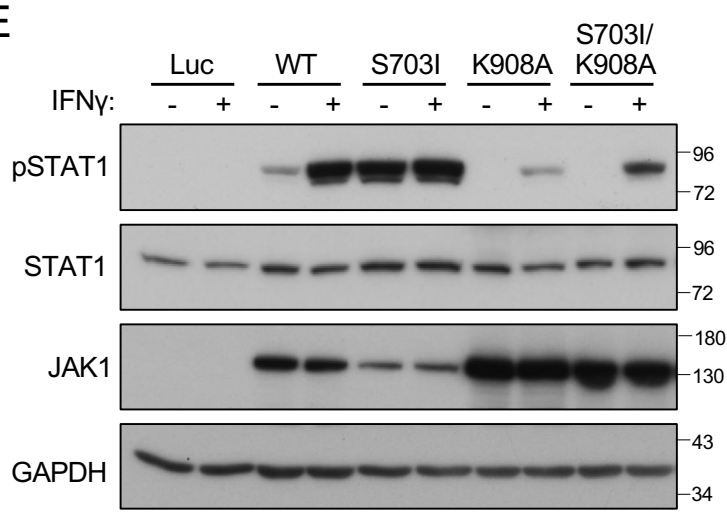

Figure 3. S703I JAK1 mediates hyperactive STAT phosphorylation by trans-activation of partnering JAKs.

(A) Immunoprecipitation of JAK1 from JAK1-transduced U4C cells and western blotting for total phosphorylation (4G10) after stimulation with IFN $\alpha(100 \mathrm{IU} / \mathrm{mL})$ for 15 minutes. (B) Immunoprecipitation of JAK2 from JAK1-transduced U4C cells and western blotting for phosphorylation at the activation loop after stimulation with IFN $\alpha(100 \mathrm{IU} / \mathrm{mL})$ for 15 minutes. (C) Immunoprecipitation of TYK2 from patient-derived B-EBV cells and western blotting for phosphorylation at the activation loop after stimulation with IFNy $(0.1 \mathrm{ng} / \mathrm{mL})$ for 15 minutes. (D). Transduction of U4C cells with catalytically inactivated JAK1 (K908A), WT JAK1, S703I JAK1, or double mutant JAK1 (K908A S703I), followed by stimulation with IFN $\alpha$ (1000 $\mathrm{IU} / \mathrm{mL})$ or (E) IFNy $(1.0 \mathrm{ng} / \mathrm{mL})$.

expressed canonical defining markers, but it had not undergone such expansion.

We then performed phospho-CyTOF to analyze the phosphorylation of all STATs downstream from JAK1 in all major immune cells of whole blood. Given the ubiquitous expression and diverse signaling capabilities of JAK1, as well as the basal activity of S703I observed in vitro, we hypothesized that all STATs within all immune cells would be hyperphosphorylated at baseline. Indeed, heightened STAT phosphorylation was observed, but not universally. Certain immune subsets, but not others, exhibited high basal phosphorylation of specific STATs (Figure 4C). For example, granulocytes from the patient displayed baseline STAT1 phosphorylation, but not
STAT3 phosphorylation, whereas T cells displayed basal STAT3 phosphorylation but not STAT1 phosphorylation. STAT6 phosphorylation, however, was not significantly upregulated in any immune subset except B cells.

To evaluate the functional consequences of the observed basal phosphorylation, we assessed the expression of downstream genes from bulk PBMCs. We detected elevated expression of many downstream pSTAT target genes, including IFIT1 and SIGLEC1 (Figure S4A). In addition, we tested nonhematopoietic tissues for baseline STAT phosphorylation by immunohistochemistry. In skin, gastrointestinal and renal biopsies we detected phosphorylated STAT1 and STAT3 (Figure S4B). This 
A
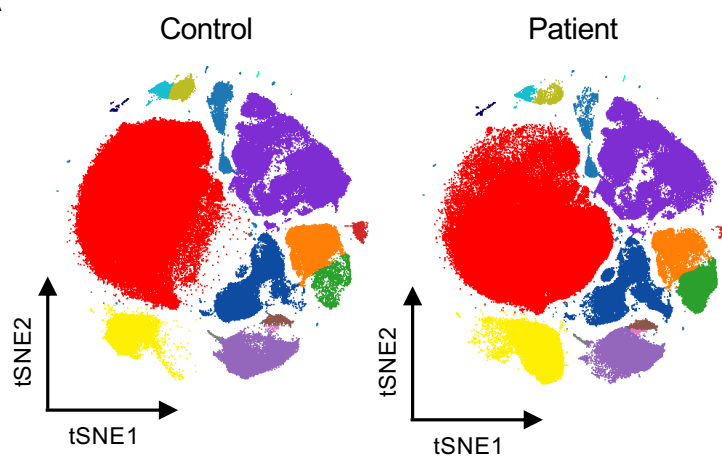

C
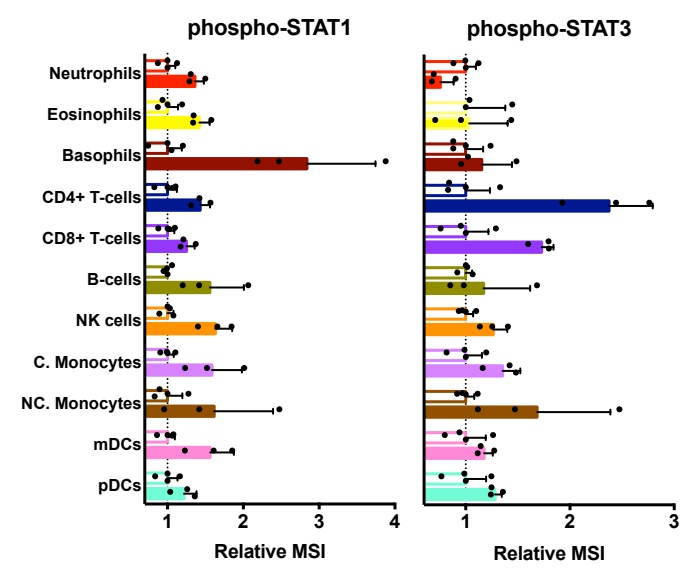

Healthy Controls $(n=4)$
B

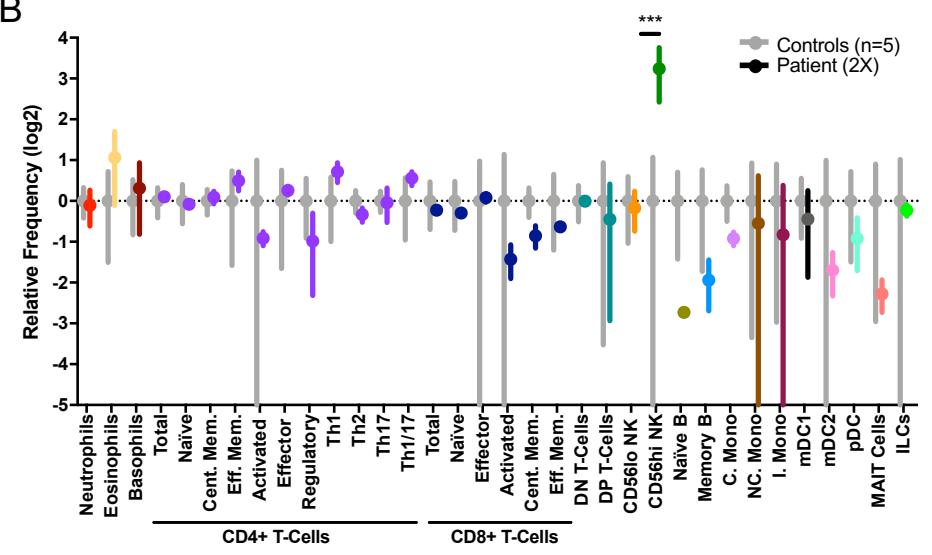

Figure 4. CyTOF analysis reveals cytokine-, STAT- and cell-type-specific gain-of-function.

(A) Representative tSNE plots generated from immunophenotyping CyTOF data of whole blood. (B) Manually gated CyTOF populations from whole blood of five separate healthy controls and the patient on two separate occasions (2X) were quantified as percent of single cells and expressed as relative frequency (patient/controls). Grey bars indicating mean with standard deviation of healthy donors, and colored bars indicating mean with standard deviation of patient. Multiple ttests performed correcting for multiple comparisons using the Holm-Sidak method. ${ }^{\star \star *} P<0.001$. (C) Relative MSI (patient/control) of phospho-STAT staining from intracellular phospho-CyTOF of whole blood from four healthy donors $(\mathrm{n}=4)$ and the patient on three separate occasions (3X). (D) Ex vivo stimulation with IFN $\alpha(100 \mathrm{IU} / \mathrm{mL}), \mathrm{IL}-2(50 \mathrm{ng} / \mathrm{mL})$ and IL-4 $(50 \mathrm{ng} / \mathrm{mL})$ for 15 minutes. Color intensity indicates the log2 fold-change in MSI between patient and healthy control $(n=1)$.

basal phosphorylation was observed in the apparent absence of any overt increase in circulating JAKSTAT cytokine levels (Figure S4C), suggesting that intrinsic S703I JAK1 activity drives this process, consistent with our in vitro results (Figure 2B-F).

\section{Ex vivo cytokine stimulation generates novel pathways in patient cells}

Whole blood was then stimulated ex vivo with a series of cytokines that engage JAK1 with various cytokine receptors, JAK partners and downstream STAT targets. In response to IFNa or IL-2, patient leukocytes hyper-phosphorylated STAT1 and STAT3, or STAT5, respectively (Figure 4D, represented differently in Figure S5A-C). Of note, NK cells exhibited the strongest response to IFNa and IL-2. By contrast, STAT6 phosphorylation in response to IL-4 was similar to that in healthy control cells, as seen in the baseline STAT6 data (Figure 4D, S5C). The same differential response was noted in patient B-EBV cells (Figure S5D-E). Interestingly, CyTOF analysis revealed that both $\mathrm{IL}-4$ and $\mathrm{IL}-2$ induced the phosphorylation of STAT1 in patient cells, contrasting with the canonical signaling cascade induced by these 
cytokines (Fig 4D). This non-canonical response suggests that S703I confers promiscuity onto JAK1, allowing it to transverse traditional signaling axes and establish novel pathways. Together, these results indicate that S703I JAK1 is a gain-of-function mutation ex vivo, displaying both unexpected pathway promiscuity and cell-type specificity in the activation of STAT signaling.

\section{Custom scRNAseq maps cellular distribution and transcriptomic signatures of S703I JAK1}

To follow up on these striking differences across the patient's cell types, we performed single cell RNA sequencing of patient PBMCs. We aimed to specifically map and evaluate the impact of S703I JAK1 across immune cell subsets by relating single cell-resolution gene expression patterns to cell type and, given the mosaicism, JAK1 genotype. However, sequence data from droplet-based scRNA-Seq platforms are typically restricted to 5' or 3' transcript regions and therefore do not include coverage of the S703I site (c.2108) in JAK1 mRNA (Figure S6A). Therefore, using custom barcode microbeads and a modified library preparation procedure, we adapted the inDrop scRNA-Seq methodology (Klein et al., 2015; Zilionis et al., 2017) to target exon 16 (containing the S703I site) of JAK1 in addition to standard mRNA 3' regions (Figure S6B, details in Methods). We used JAK1-targeted inDrop scRNASeq to analyze patient PBMCs, and concurrently processed the same sample on the 10X Genomics Chromium platform (Figure S6B). Clustering and manual annotation of cell types based on gene expression patterns distinguished the expected PBMC populations, with relative frequencies consistent with the corresponding CyTOF analyses (Figure 5A, S6C). Again, we observed a relative increase in CD56 ${ }^{\text {hi }} \mathrm{NK}$ cell frequency. Using sequence data from JAK1-targeted libraries, we assigned putative per cell JAK1 genotypes (based on transcript sequences) to a subset of PBMCs for which transcript and read depths were sufficient in the JAK1-targeted inDrop dataset (Figure S6D-E). Within this subset, we found that the mutant allele was not evenly distributed across immune cell-type clusters (Figure 5B-C). For example, B cells and monocytes mostly carried WT transcript, whereas $69 \%$ of CD56 ${ }^{\text {hi }}$ NK cells contained JAK1 mRNA with the S703I mutation. This variable distribution may reflect differences in the intrinsic tolerance to the mutation in these cell types, which is consistent with the expansion of CD56 ${ }^{\text {hi }}$ NK cell subset and the higher levels of JAK1 expression in NK cells than in other cell types (Figure S6F).

We next performed differential gene expression analysis, comparing patient cells expressing the WT and mutant alleles of JAK1. Although this analysis was constrained by a limited number of cells for which transcript-level genotype information was available, the intrasample comparison can be conducted on an inherently isogenic background with identical exposure history. We detected statistically significant differences in ISG expression for IFI44L and for the entire ISG gene set between WT and mutant monocytes (Figure 5D-E). These data suggest that tolerance to S703I may differ between cell types and confirm the cell-intrinsic nature of the gain-of-function described above.

\section{Biased expression of JAK1 alleles}

Given the mosaicism of a heterozygous mutation, we expected to observe some cells (i.e. homozygous WT $J A K 1)$ containing only WT JAK1 transcripts and others (i.e. heterozygous S703I JAK1) containing both WT and S703I JAK1 transcripts. Surprisingly, we found that expression of the two alleles seemed almost mutually exclusive, as very few cells expressed both transcripts, as opposed to the $\sim 50 \%$ expected given our genomic estimates (Figure 5F left panel, Figure 1D). By contrast, other genes from our dataset containing heterozygous variants exhibited the expected biallelic distribution (Figure $5 \mathrm{~F}$ right panel). This result suggests that JAK1 may be subject to monoallelic bias, a pattern that has only recently been recognized in a fraction of the autosomal transcriptome (Gimelbrant et al., 2007; Jeffries et al., 2012; Deng et al., 2014; Borel et al., 2015). We further tested this hypothesis of biased allele expression by sorting single cells from healthy donor PBMCs heterozygous for a synonymous SNP (rs2230587) of $J A K 1$, adjacent to S703I. qPCR of isolated RNA with allele-specific probes revealed that relative expression levels of the two alleles were not normally distributed, but rather biased to one allele or the other (Figure 5G), unlike in a control gene (Figure 5H). Query of publicly available murine expression data revealed a similar allele restriction for JAK1, which, at least in mice, remains fixed over time (Savova, Patsenker and Gimelbrant, 2016). Whether the observed phenomenon in this patient represents transcriptional bursting or mitotically stable monoallelic expression, and the potential impact on immune dysfunction, remains to be fully determined. In either sense, these data, perhaps, indicate departure from the classic genetic interpretation of heterozygosity and allow for a shift in understanding of genetic penetrance of disease. 
bioRxiv preprint doi: https://doi.org/10.1101/807669; this version posted October 17,2019 . The copyright holder for this preprint (which was not certified by peer review) is the author/funder, who has granted bioRxiv a license to display the preprint in perpetuity. It is made available under aCC-BY-ND 4.0 International license.

A

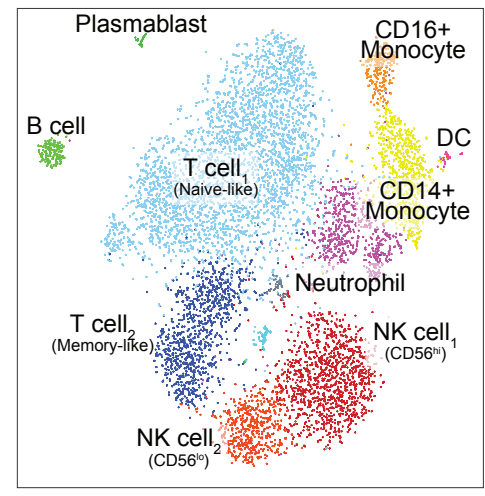

D

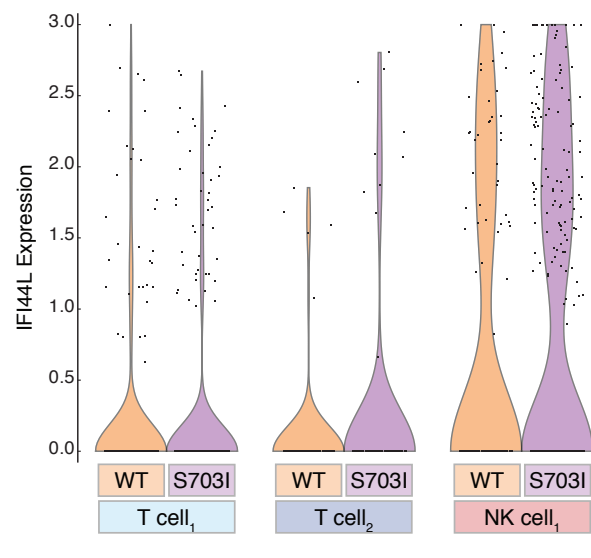

F

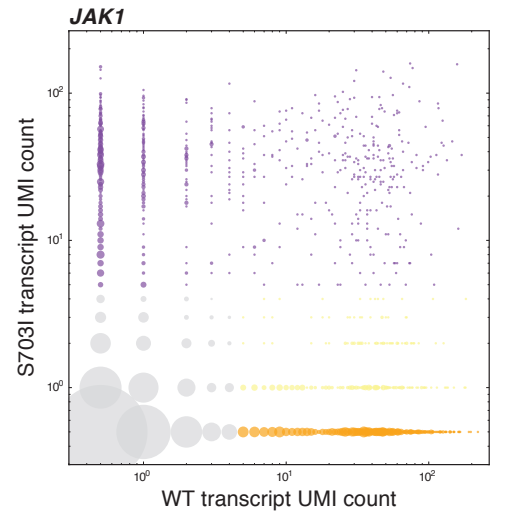

B

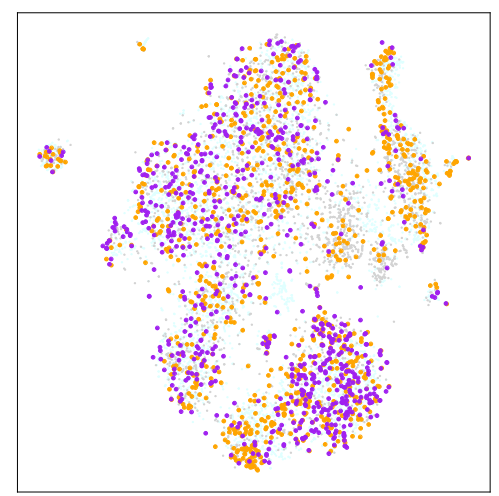

WT JAK1 —S703I JAK1
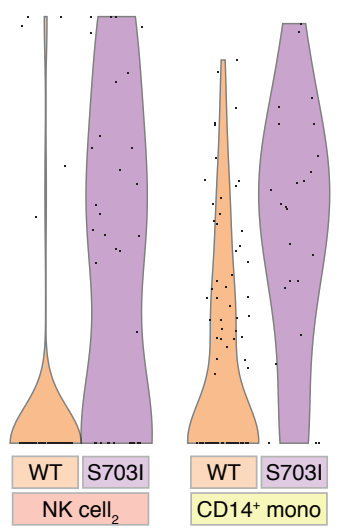

G

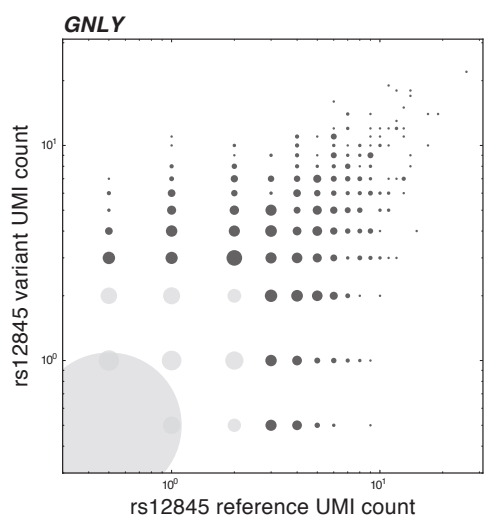

C

E

$\mathrm{H}$
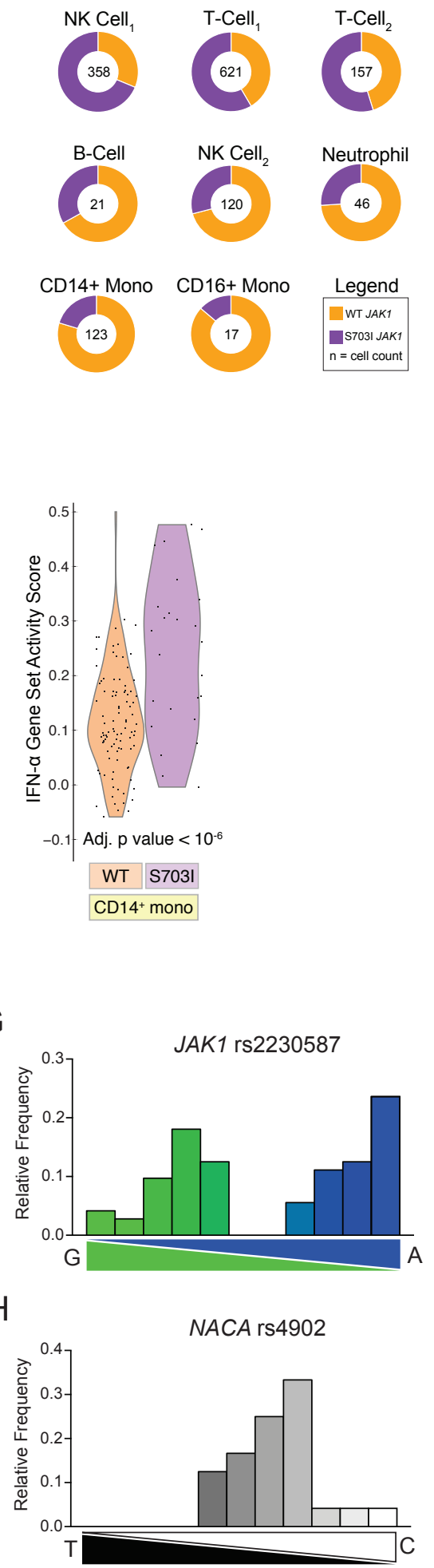

Figure 5. Custom single-cell-RNA sequencing maps JAK1 allele distribution, transcriptomic impact and expression patterns.

(A) tSNE plots and cell type assignments from single cell RNA sequencing of patient PBMCs with an inDrop platform adapted to target the mutant JAK1 transcript. $n=4763$ cells. (B) tSNE plots representing the subset of cells with sufficient JAK1 counts to be assigned putative JAK1 genotypes (based on transcript sequences). Cells in which any mutant transcript was detected above empiricallydetermined thresholds were assigned 'S703I JAK1' (purple) while cells with only WT transcript detected were assigned 'WT JAK1' (orange). (C) Doughnut charts quantifying allele distribution in cells meeting genotyping criteria (cell count in the center), as in panel B. (D) Expression of the interferon stimulated gene IFI44L, a statistically significant differentially expressed gene in the comparison of WT JAK1 and S703I JAK1 genotyped cells. (E) Gene set scores for IFN $\alpha$ signaling in CD14+ monocytes (F) Number of unique transcripts detected per cell for the WT or S703I JAK1 allele (left) or a control variant GNLY rs12845 (right). Bubble size indicates number of cells. Color coding indicates cells containing: S703I JAK1 (purple), WT JAK1 without S703I JAK1 (orange), WT JAK1 with S703I JAK1 detected below threshold (yellow), or insufficient transcripts counts (gray). (G) Transcript genotyping of JAK1 rs2230587 from healthy control PBMCs $(n=96)$ by single cell qPCR with allele-specific probes. Histogram represents relative frequency of cells expressing binned allele ratios as quantified by oligonucleotide standards. (I) Single cell qPCR transcript genotyping of control gene NACA (rs4902). 
Clinical and biological rescue with tofacitinib

Having identified JAK1 hyperactivity as the putative driver of clinical disease in the patient, we then considered the use of JAK inhibitors for the clinical treatment of this patient. We first compared the ability of the two FDA-approved inhibitors available at the time to reduce basal STAT phosphorylation in S703Itransduced U4C cells. Despite its lower relative potency against JAK1, tofacitinib inhibited STAT phosphorylation in a comparable dose response to ruxolitinib (Figure 6A), again reflecting the independence of JAK1 catalysis and STAT hyperphosphorylation. Similar results were obtained with patient-derived B-EBV cells, which, unlike U4Cs, express JAK3 (Figure 6B). Next, we treated patient blood ex vivo with the two compounds at equimolar doses that mimic physiological dosing (Chen et al., 2014; Krishnaswami et al., 2014; Lamba et al., 2016), and we further assessed the inhibition of IFNa stimulation. Analysis of phospho-STAT inhibition across whole blood immune subsets by phosphoCyTOF revealed that tofacitinib attenuated the response more potently than ruxolitinib in nearly all cell types (Figure 6C).

Following these extensive functional studies, we treated the patient with low-dose tofacitinib $(5 \mathrm{mg}$ daily). Remarkably, within eight weeks, circulating inflammatory markers (ESR and C-reactive protein) normalized (Figure 6D-E). Significant improvement in dermatitis followed, both grossly and histologically (Figure 6F). By six months, the patient reported complete resolution of gastrointestinal symptoms (decrease in modified PUCAI from 35-50 initially to $0)$. Biopsy of colonic tissue revealed restoration of crypt architecture and complete resolution of eosinophilic infiltrates (Figure 6G). Twelve months after the initiation of treatment, the patient remains stable and is awaiting re-transplantation.

Finally, we confirmed the pharmacological rescue of JAK hyperactivity in the patient's cells after tofacitinib treatment. RNA isolated from PBMCs revealed that the expression of ISGs, which was significantly elevated before treatment, progressively declined to normal levels (Figure $6 \mathrm{H}$ ). Mass cytometry analysis was then performed to confirm the decrease in basal STAT phosphorylation. Drastic reductions were observed across cell types in STAT3, STAT4, STAT5 and STAT6 phosphorylation, whereas STAT1 phosphorylation levels were reduced in some, but not all cell types (Figure 6I). Overall, these results validate S703I JAK1 as the etiology in vivo of the widespread immune dysregulation, and they illustrate the striking power of precision medicine both as a life-saving approach for patients with rare diseases and as a means of discovering novel and fundamental physiological mechanisms.

\section{Discussion}

Undiagnosed disease programs have proven remarkably successful at detecting potential causative variants of disease (Splinter et al., 2018; Lee et al., 2019; Yang et al., 2019). This report demonstrates the value of in-depth study of select patients identified in these programs. Most immediately, the findings described herein directed the successful treatment of a complex immunodysregulatory disease in a highly personalized molecular fashion. More broadly, this case implicates JAK1 dysfunction in common forms of multifactorial diseases, including dermatitis, enteritis, colitis and eosinophilic disorders. These features align with the other reported JAK1 GoF mutation recently published (Del Bel et al., 2017), and together, provide strong justification for the expanding use of JAK inhibitors in these disorders (O'Shea et al., 2015; O'Shea and Gadina, 2019). Yet, to date, MN has not been recognized to involve JAK-STAT dysregulation. It logically follows that MN, the most common cause of nephrotic syndrome, may be amenable to early treatment with JAK inhibitors. In fact, baricitinib, a JAK1/JAK2 inhibitor, demonstrated recent success in a clinical trial for diabetic nephropathy, a related nephrotic syndrome (Tuttle et al., 2018).

The absence of MN in the other reported JAK1 GoF mutation (A634D) may represent important distinctions in the behavior of different mutated forms of JAK1, rather than variable penetrance of the same genetic etiology. Disruptions of the pseudokinase domain may have vastly divergent functional consequences, which can already be gleaned by comparing the JAK1 mutations identified to date: P733L and P832S (LoF) vs. A634D and S703I (GoF). Moreover, our findings indicate that GoF may not necessarily lead to the universal activation of downstream pathways, as S703I caused the hyperactivation of some pathways, but not others. Furthermore, disruption of the pseudokinase domain by $\mathrm{S} 703 \mathrm{I}$ enabled cells to respond promiscuously via non-canonical signaling pathways. Together, these findings suggest that the pseudokinase domain is not a simple "on/off" switch. This regulatory complexity may stem from the ability of the JAK1 pseudokinase to modulate the activity of JAK2 and TYK2, as demonstrated here. Consequently, careful study of each mutation is warranted, each yielding valuable information on fundamental JAK1 function. The complex regulation becomes especially important given the high incidence of oncogenic JAK mutations, as well as the expanding therapeutic use of JAK inhibitors. Regarding the latter, the evidence 
bioRxiv preprint doi: https://doi.org/10.1101/807669; this version posted October 17, 2019. The copyright holder for this preprint (which was not certified by peer review) is the author/funder, who has granted bioRxiv a license to display the preprint in perpetuity. It is made available under aCC-BY-ND 4.0 International license.

A

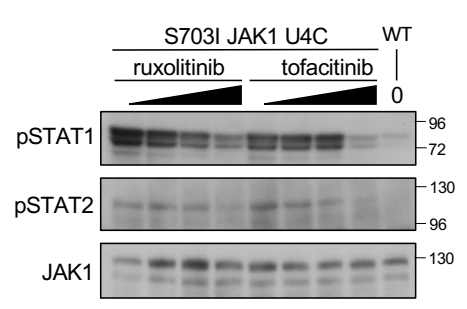

B

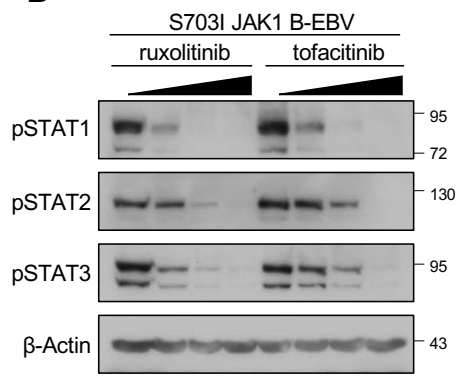

C

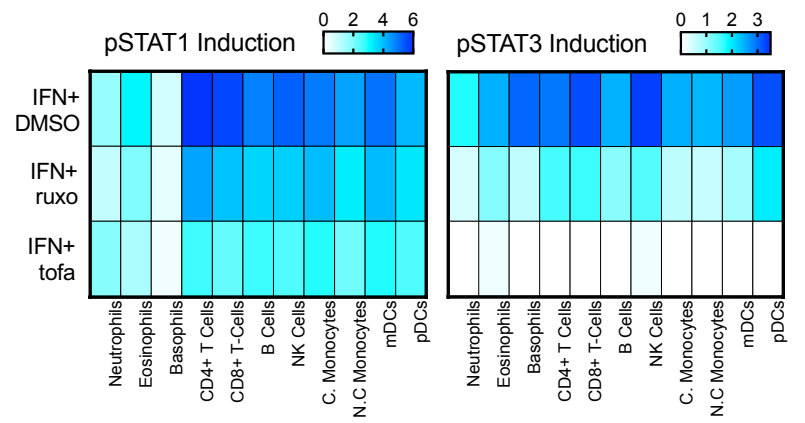

F

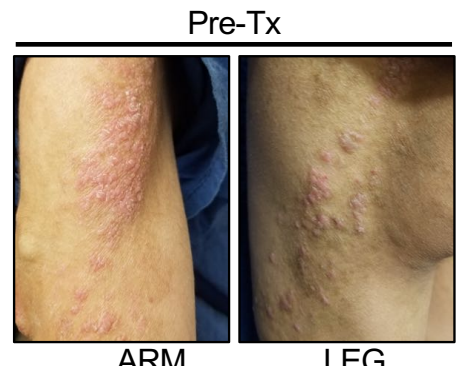

ARM

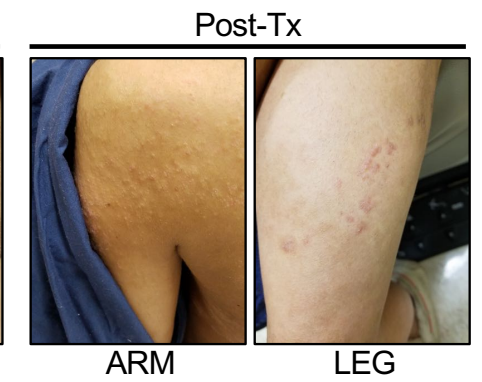

$\mathrm{H}$

MX1 mRNA

IFIT1 mRNA

SIGLEC1 mRNA
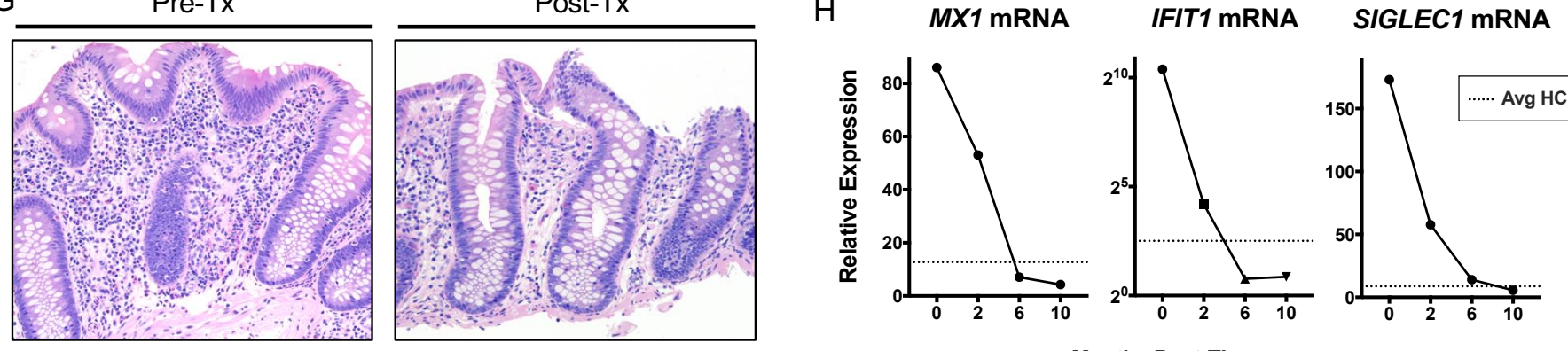

Months Post-Therapy

I

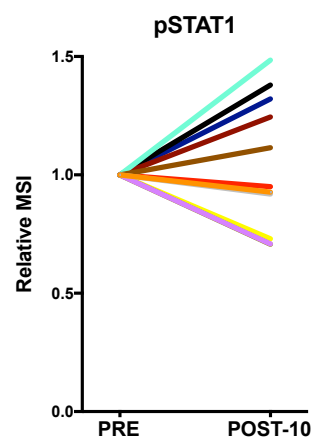

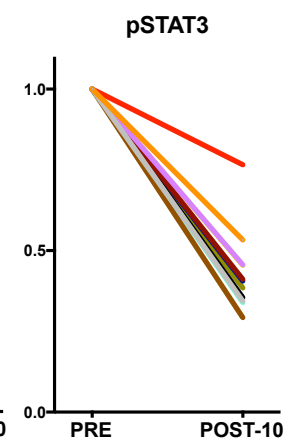
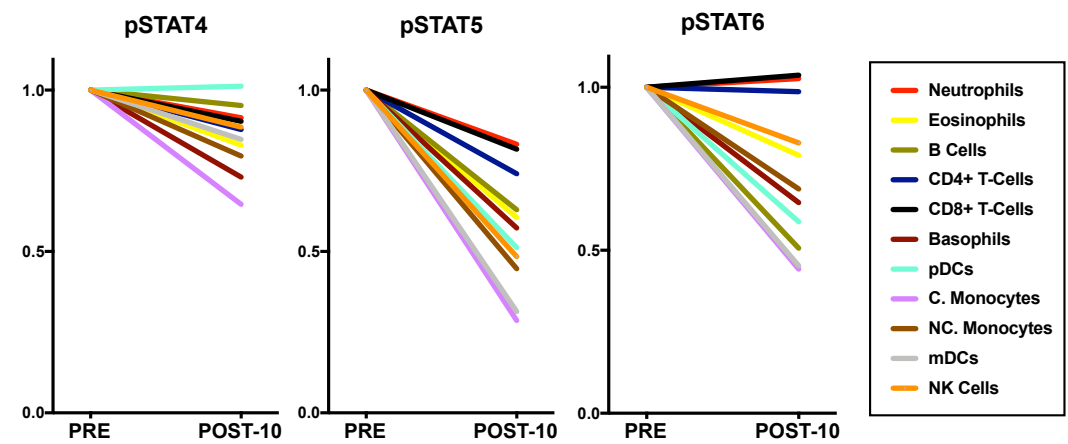

Figure 6. Treatment with tofacitinib rescues STAT hyperactivity and resolves clinical disease.

(A) In vitro assessment of JAK-inhibitor efficacy after 4-hour drug treatment for resolution of basal STAT phosphorylation in transduced U4Cs. (B) Similar analysis in patient-derived B-EBVs. (C) Ex vivo inhibition with equimolar doses (500 $\mathrm{nM}$ ) of ruxolitinib and tofacitinib for 4 hours followed by IFN $\alpha$ stimulation $(1000 \mathrm{IU} / \mathrm{mL})(\mathrm{n}=1)$. (D \& E) Acute phase reactants from peripheral blood. (F) Gross appearance of skin before and 5-months after tofacitinib initiation. (G) Colonic biopsies before and 6-months post-therapy. (H) ISG expression in RNA from bulk PBMCs isolated throughout treatment $(n=1)$. (I) Phospho-CyTOF analysis comparing relative changes in pSTAT MSI from before and 10-months post-therapy $(n=1)$. 
presented here and elsewhere (Haan et al., 2011; Li et al., 2013; Eletto et al., 2016) of the highly cooperative action of JAKs challenges the strategic wisdom of increasing the selectivity of JAK inhibitors.

Downstream from the JAK-receptor complex, S703I JAK1 upregulates STAT1, STAT2, STAT3, and STAT5 phosphorylation and, correspondingly, downstream gene transcription. We demonstrate here that this GoF occurs in a hyper-responsive fashion ex vivo, but that intrinsic signaling can occur independent of cytokine, as evidenced by basal STAT phosphorylation in the absence of a detectable increase in JAK-STAT cytokines. However, it is difficult to isolate which specific pathways cause disease, as JAK1 mediates signaling for more than twenty five cytokines (O'Shea et al., 2015). This broad immunodysregulatory disease is more likely to be driven by constitutive activity of a gamut of cytokine pathways, which have innumerable functions in autoinflammation and autoimmunity.

Nevertheless, specific immune disturbances were evident. We observed a robust upregulation of type I IFN signaling in this patient. Furthermore, in both published cases of JAK1 GoF eosinophils increase in circulation and infiltrate affected tissues. These findings are consistent with the features of atopic disease in both cases. Our high-dimensional analyses also detected a profound dysregulation of NK cells. CD56 ${ }^{\text {hi }}$ NK cells demonstrated the largest difference in frequency, exhibited the most upregulated pSTAT profile, and carried the highest percentage of mutant transcript. Consistent with their phenotype, CD56 ${ }^{\text {hi }}$ NK cells generally express high levels of JAK1, which likely potentiates the GoF effect in NK cells from the patient. This expansion likely arises from the intrinsic STAT5 activity and IL-2hyperresponsive signaling of S703I JAK1, which is known to expand CD56hi NK cells (Poli et al., 2009). It is worth noting that long-term clinical treatment with IL-2 (Caligiuri et al., 1993) or IFN $\beta$ (Saraste, Irjala and Airas, 2007), disturbances that phenocopy JAK1 GoF, also lead to $\mathrm{CD}^{\mathrm{hi}}{ }^{\mathrm{hi}}$ expansions. Interestingly, although tofacitinib clearly reduced transcriptomic and pSTAT inflammatory signatures and resolved clinical disease, we did not observe reversal of the CD56hi NK cell enrichment. The pharmacokinetic profile of tofacitinib may be such that it decreases the function of these cells, but not their number. In any case, it is apparent that persistent JAK1-mediated cytokine signaling has critical consequences on NK cells.

Finally, this study is among the first to demonstrate somatic mosaicism underlying immunologic disease (Holzelova et al., 2004; Saito et al., 2005; Wolach et al., 2005; Tanaka et al., 2011; Liu et al., 2014; de Inocencio et al., 2015). Mosaicism remains an underappreciated feature of monogenic disorders. Recent studies indicate that undetected somatic variation is incredibly common (predicted $10^{16}$ base substitutions), and that up to $6.5 \%$ of de novo mutations identified to date are actually mosaic (Acuna-Hidalgo et al., 2015). Mosaicism becomes especially important as we incorporate WES into the future of clinical medicine. Potential disease-causing mutations may be overlooked without careful consideration of the tissue site genotyped, loci with low frequency variants, and the poor sensitivity of classic Sanger sequencing. Additionally, asymmetric clinical manifestations like those observed here (leg length discrepancy and dermatitis along lines of embryological migration) should prompt suspicions of mosaicism and guide genetic analysis.

The analyses described here have only recently become possible with technological advances in single-cell assays. Here, by adapting the inDrop single cell RNA-seq platform, we implement a novel approach for the detection and analysis of a specific mutation within a transcript region not readily accessible by other methods. The resulting single cell resolution data allowed us to determine mutant allele frequency in different cell populations, identify mutation-associated gene expression patterns, and unexpectedly, to observe allelic bias in transcription of $J A K 1$. This bias is consistent with recent evidence of widespread transcriptional bursting and monoallelic expression of autosomal genes (Gimelbrant et al., 2007; Jeffries et al., 2012; Borel et al., 2015; Reinius and Sandberg, 2015). Importantly, this is the first report of monoallelic expression of a mutated gene. Biased allelic expression, in conjunction with mosaicism, may prove an important point of focus for future genetic studies of variable penetrance, affected carriers and undiagnosed disease.

\section{Acknowledgements}

We thank Adeeb Rahman, Brian Lee, Kevin Tuballes and Laura Walker from Human Immune Monitoring Center and Rachel Brody from the Biorepository and Pathology CoRE at the Icahn School of Medicine for their technical assistance and guidance in experimental design. This research was supported by National Institute of Allergy and Infectious Diseases Grants R01Al127372, R21 Al134366 and R21Al129827, and funding from the March of Dimes, awarded to DB. Research in the Mace laboratory was supported by JSO R01Al120989 and research in the Rosenberg laboratory by DP5 OD012142. CG was supported by T32 training grant 5T32HD075735-07. 


\section{Author contributions}

CG designed and performed most of the experiments, analyzed the data and wrote the manuscript. JC analyzed all scRNAseq data and edited the manuscript. SB generated cell lines and processed whole blood experiments. GE analyzed sequencing data, performed ddPCR and edited the manuscript. JM performed the tissue processing for ddPCR. SU and $\mathrm{RC}$ designed the platform and carried out the custom scRNAseq experiments. EM and JO performed the NK cell experiments and analysis. BW, MM, BG helped to design the experiments and analyze data. LJ, DD, RP, JS performed clinical analyses and reports. BR designed the custom scRNAseq, analyzed the data and edited the manuscript. BW, MM, JO, BG helped to design the experiments and analyze data. DB helped to design the experiments and analyze data, supervised the work and wrote the manuscript. All authors commented on the manuscript.

\section{Data Availability}

The raw data for experiments performed will be made available, including the PCR, multiplex ELISA, mass cytometry, flow cytometry, scRNAseq data shown in Figure 1D, 2C, 4, 5, 6C-I, S1E, S3, S4A, S4C, S5A-C, S6, S7. Web links will be made available for large files, including fcs files and scRNAseq gene $x$ cell matrices. For privacy concerns of the study participants, the complete data files from whole exome sequencing will be restricted to the variants in Table 1.

\section{Code Availability}

Python scripts and associated Seurat code (R-based) used for the tailored analysis of JAK1-specific scRNAseq will be made available upon publication of the manuscript. Contact dusan.bogunovic@mssm.edu.

\section{References}

Acuna-Hidalgo, R. et al. (2015) 'Post-zygotic Point Mutations Are an Underrecognized Source of de Novo Genomic Variation', American Journal of Human Genetics. The American Society of Human Genetics, 97(1), pp. 67-74. doi: 10.1016/j.ajhg.2015.05.008.

Babon, J. J. et al. (2014) 'The molecular regulation of Janus kinase (JAK) activation', Biochemical Journal, 462(1), pp. 1-13. doi: 10.1042/BJ20140712.

Del Bel, K. L. et al. (2017) 'JAK1 gain-of-function causes an autosomal dominant immune dysregulatory and hypereosinophilic syndrome', Journal of Allergy and Clinical Immunology, 1(June). doi: 10.1016/j.jaci.2016.12.957.
Borel, C. et al. (2015) 'Biased allelic expression in human primary fibroblast single cells', American Journal of Human Genetics. The American Society of Human Genetics, 96(1), pp. 70-80. doi: 10.1016/j.ajhg.2014.12.001.

Caligiuri, M A et al. (1993) 'Selective modulation of human natural killer cells in vivo after prolonged infusion of low dose recombinant interleukin 2 . Find the latest version':, Journal of Clinical Investigation, 91(1), pp. 123-132.

Chen, X. et al. (2014) 'Pharmacokinetics and pharmacodynamics of orally administered ruxolitinib (INCB018424 phosphate) in renal and hepatic impairment patients', Clinical Pharmacology in Drug Development, 3(1), pp. 3442. doi: $10.1002 / \mathrm{cpdd} .77$.

Deng, Q. et al. (2014) 'Single-Cell RNA-Seq Reveals Dynamic, Random Monoallelic Gene Expression in Mammalian Cells', 343(January). doi: 10.1126/science.1245316.

Dupuis, S. et al. (2001) 'Impairment of Mycobacterial But Not Viral Immunity by a Germline Human STAT1 Mutation', Science, 293(5528), pp. 300303. doi: 10.1126/science. 1061154.

Eletto, D. et al. (2016) 'Biallelic JAK1 mutations in immunodeficient patient with mycobacterial infection', Nature Communications. Nature Publishing Group, 7, p. 13992. doi: 10.1038/ncomms13992.

Etheridge, S. L. et al. (2014) 'A novel activating, germline JAK2 mutation, JAK2R564Q, causes familial essential thrombocytosis', Blood, 123(7), pp. 1059-1068. doi: 10.1182/blood-2012-12473777.

Gimelbrant, A. et al. (2007) 'Widespread monoallelic expression on human autosomes', Science, 318(5853), pp. 1136-1140. doi: 10.1126/science.1148910.

Haan, C. et al. (2011) 'Jak1 has a dominant role over Jak3 in signal transduction through yc-containing cytokine receptors', Chemistry and Biology, 18(3), pp. 314-323. doi: 10.1016/j.chembiol.2011.01.012.

Hambleton, S. et al. (2013) 'STAT2 deficiency and susceptibility to viral illness in humans', Proceedings of the National Academy of Sciences, 110(8), pp. 3053-3058. doi: 10.1073/pnas.1220098110.

Holland, S. M. et al. (2007) 'STAT3 Mutations in the Hyper-IgE Syndrome', New England Journal of Medicine, 357(16), pp. 1608-1619. doi: 10.1056/NEJMoa073687.

Holzelova, E. et al. (2004) 'Autoimmune Lymphoproliferative Syndrome with Somatic Fas Mutations', New England Journal of Medicine, 
bioRxiv preprint doi: https://doi.org/10.1101/807669; this version posted October 17, 2019. The copyright holder for this preprint (which was not certified by peer review) is the author/funder, who has granted bioRxiv a license to display the preprint in perpetuity. It is made available under aCC-BY-ND 4.0 International license.

351(14), pp. 1409-1418. doi: 10.1056/NEJMoa040036.

de Inocencio, J. et al. (2015) 'Somatic NOD2 mosaicism in Blau syndrome', Journal of Allergy and Clinical Immunology, 136(2), pp. 484-487.e2. doi: 10.1016/j.jaci.2014.12.1941.

Jeffries, A. R. et al. (2012) 'Stochastic choice of allelic expression in human neural stem cells', Stem Cells, 30(9), pp. 1938-1947. doi: 10.1002/stem.1155.

Klein, A. M. et al. (2015) 'Droplet barcoding for singlecell transcriptomics applied to embryonic stem cells.', Cell, 161(5), pp. 1187-1201. doi: 10.1016/j.cell.2015.04.044.

Kofoed, E. M. et al. (2003) 'Growth Hormone Insensitivity Associated with a STAT5b Mutation', New England Journal of Medicine, 349(12), pp. 1139-1147. doi: 10.1056/NEJMoa022926.

Krishnaswami, S. et al. (2014) 'Pharmacokinetics of tofacitinib, a janus kinase inhibitor, in patients with impaired renal function and end-stage renal disease', Journal of Clinical Pharmacology, 54(1), pp. 46-52. doi: 10.1002/jcph.178.

Lamba, M. et al. (2016) 'Extended-Release OnceDaily Formulation of Tofacitinib: Evaluation of Pharmacokinetics Compared With ImmediateRelease Tofacitinib and Impact of Food', Journal of Clinical Pharmacology, (March), pp. 1362-1371. doi: $10.1002 / j c p h .734$.

Lee, H. et al. (2019) 'Clinical Exome Sequencing for Genetic Identification of Rare Mendelian Disorders', Journal of American Medical Association, 90095(18), pp. 1880-1887. doi: 10.1001/jama.2014.14604.

Li, Z. et al. (2013) 'Two Rare Disease-Associated Tyk2 Variants Are Catalytically Impaired but Signaling Competent', The Journal of Immunology, 190(5), pp. 2335-2344. doi: 10.4049/jimmunol.1203118.

Liu, Y. et al. (2014) 'Activated STING in a Vascular and Pulmonary Syndrome', New England Journal of Medicine, 371(6), pp. 507-518. doi: 10.1056/NEJMoa1312625.

Macchi, P. et al. (1995) 'Mutations of Jak-3 gene in patients with autosomal severe combined immune deficiency (SCIO)', Nature, 377(September), pp. 65-68.

Mead, A. J. et al. (2012) 'Germline JAK2 Mutation in a Family with Hereditary Thrombocytosis', New England Journal of Medicine, 366(10), pp. 967969. doi: 10.1056/NEJMc1200349.

Minegishi, Y. et al. (2006) 'Human Tyrosine Kinase 2 Deficiency Reveals Its Requisite Roles in Multiple Cytokine Signals Involved in Innate and Acquired Immunity', Immunity, 25, pp. 745-755. doi: 10.1016/j.immuni.2006.09.009.

Minegishi, Y. et al. (2007) 'Dominant-negative mutations in the DNA-binding domain of STAT3 cause hyper-IgE syndrome', Nature, 448(August). doi: 10.1038/nature06096.

Moore, K., Persaud, T. and Torchia, M. (2015) Before We Are Born.

O'Shea, J. J. et al. (2015) 'The JAK-STAT Pathway: Impact on Human Disease and Therapeutic Intervention', Annual Review of Medicine, 66(1), pp. 311-328. doi: 10.1146/annurev-med-051113024537.

O'Shea, J. J. and Gadina, M. (2019) 'Selective Janus kinase inhibitors come of age', Nature Reviews Rheumatology, pp. 2-3. doi: 10.1038/s41584-0180155-9.

Poli, A. et al. (2009) 'CD56 bright natural killer (NK) cells: an important NK cell subset', Immunology, 126(4), pp. 458-465. doi: 10.1111/j.13652567.2008.03027.x.

Reinius, B. and Sandberg, R. (2015) 'Random monoallelic expression of autosomal genes: Stochastic transcription and allele-level regulation', Nature Reviews Genetics. Nature Publishing Group, 16(11), pp. 653-664. doi: 10.1038/nrg3888.

Russell, S. M. et al. (1995) 'Mutation of Jak3 in a Patient with SCID: Essential Role of Jak3 in Lymphoid Development', Science, 270(4), pp. 797-801.

Saito, M. et al. (2005) 'Somatic mosaicism ofCIAS1 in a patient with chronic infantile neurologic, cutaneous, articular syndrome', Arthritis \& Rheumatism, 52(11), pp. 3579-3585. doi: 10.1002/art.21404.

Saraste, M., Irjala, H. and Airas, L. (2007) 'Expansion of CD56Bright natural killer cells in the peripheral blood of multiple sclerosis patients treated with interferon-beta', Neurological Sciences, 28(3), pp. 121-126. doi: 10.1007/s10072-007-0803-3.

Savova, V., Patsenker, J. and Gimelbrant, A. A. (2016) 'dbMAE: the database of autosomal monoallelic', Nucleic Acids Research, 44(October 2015), pp. 753-756. doi: 10.1093/nar/gkv1106.

Splinter, K. et al. (2018) 'Effect of Genetic Diagnosis on Patients with Previously Undiagnosed Disease', New England Journal of Medicine, 379(22), pp. 2131-2139. doi: 10.1056/NEJMoa1714458.

Tanaka, N. et al. (2011) 'High incidence of NLRP3 somatic mosaicism in patients with chronic infantile neurologic, cutaneous, articular syndrome: Results of an international multicenter collaborative study', Arthritis \& Rheumatism, 63(11), pp. 3625-3632. doi: 10.1002/art.30512.

Tuttle, K. R. et al. (2018) 'JAK1/JAK2 inhibition by 
bioRxiv preprint doi: https://doi.org/10.1101/807669; this version posted October 17,2019 . The copyright holder for this preprint (which was not certified by peer review) is the author/funder, who has granted bioRxiv a license to display the preprint in perpetuity. It is made available under aCC-BY-ND 4.0 International license.

baricitinib in diabetic kidney disease: results from a

Phase 2 randomized controlled clinical trial', Nephrology Dialysis Transplantation, pp. 1-10. doi: $10.1093 / \mathrm{ndt} / \mathrm{gf} \times 377$.

Wolach, B. et al. (2005) 'Unusual late presentation of $X$-linked chronic granulomatous disease in an adult female with a somatic mosaic for a novel mutation in CYBB', Blood, 105(1), pp. 61-66. doi: 10.1182/blood-2004-02-0675.

Yang, Y. et al. (2019) 'Molecular Findings Among Patients Referred for Clinical Whole-Exome Sequencing', Journal of American Medical Association, 312(18), pp. 1870-1879. doi: 10.1001/jama.2014.14601.

Zilionis, R. et al. (2017) 'Single-cell barcoding and sequencing using droplet microfluidics', Nature Protocols. Nature Publishing Group, 12(1), pp. 4473. doi: 10.1038/nprot.2016.154. 